\title{
Inhalt
}

Vorwort - vii

Autorenverzeichnis - ix

Teil I Das Fetale Alkoholsyndrom und seine Diagnose

1 Das Syndrom - 3

1.1 Die Entdeckung des Syndroms - 3

$1.2 \quad$ Historische Entwicklung in den USA und Kanada - 4

1.3 Die Entwicklung in Europa und Deutschland - 5

1.4 Inzidenz und Prävalenz des FASD $\longrightarrow 7$

2 Diagnostik des Fetalen Alkoholsyndroms - 10

2.1 Definition -10

2.2 Das klinische Bild, historische Anmerkungen - 13

2.3 Warum ist die Diagnose so schwierig zu stellen? - 15

$\mathbf{2 . 4}$ Leitlinienentwicklungen zur Diagnose $\mathbf{1 7}$

3 Der 4-Digit Diagnostic Code -22

$3.1 \quad$ Einleitung -22

3.2 Die Wachstumsstörung -25

3.3 Faziale Dysmorphie - 28

3.4 Schädigung des zentralen Nervensystems - 35

3.5 Alkoholexposition in der Schwangerschaft -39

4 Die diagnostischen Besonderheiten in den einzelnen

\section{Lebensabschnitten -45}

4.1 Postnatal und frühe Kindheit -47

4.2 Kindheit und Vorschulalter $-\mathbf{5 1}$

4.3 Schulalter $-\mathbf{5 8}$

$4.4 \quad$ Jugend und Adoleszenz -64

4.5 Erwachsenenalter („,FAS adult“) -70

$5 \quad$ Begleitstörungen beim FASD -73

5.1 FASD und Schlafstörungen -73

5.2 FASD und organische Fehlbildungen -79

5.3 FASD und Differenzialdiagnosen - $\mathbf{8 8}$

5.4 FASD und psychiatrische Erkrankungen - $\mathbf{9 0}$

5.5 FASD und ADHS -92 
Teil II Wissenschaftliche Grundlagen zum Fetalen Alkoholsyndrom

6 Epidemiologie der Fetalen Alkoholspektrumstörungen - 101

6.1 Alkoholkonsum in Deutschland - 101

6.2 „Moderates Trinken“ 105

7 Teratogenität des Alkohols - 112

7.1 Allgemeines $\mathbf{1 1 2}$

7.2 Tierversuche zur Teratogenität von Alkohol - behavioral teratology -114

7.3 Zeitpunkt der Alkohol-Exposition — $\mathbf{1 1 5}$

$8 \quad$ Nikotin und illegale Drogen in der Schwangerschaft -118

8.1 Rauchen in der Schwangerschaft - $\mathbf{1 1 8}$

8.2 Kokain und Heroin in der Schwangerschaft $-\mathbf{1 2 0}$

8.3 Das „Crack-Baby“- 121

8.4 Cannabis/Marihuana - $\mathbf{1 2 2}$

8.5 Polydrug-Missbrauch in der Schwangerschaft -124

9 Biomarker zum Nachweis mütterlichen Alkoholkonsums in der Schwangerschaft -126

9.1 Direkte und indirekte Biomarker — 126

$10 \quad$ Neuropathologische Aspekte und Pathogenese der FASD 128

$10.1 \quad$ Einleitung -128

10.2 Frühe neuropathologische Befunde $-\mathbf{1 2 9}$

10.3 Tierexperimentelle Forschung zur Pathogenese - 130

10.4 Bildgebende Verfahren - 138

10.5 Störungen des Hirnmetabolismus $\mathbf{1 4 0}$

11 Genetische und epigenetische Aspekte beim FASD 143

11.1 Genetik - 143

11.2 Epigenetik -144

Teil III Langzeitstudien und das Fetale Alkoholsyndrom

12 Langzeitstudien zum Fetalen Alkoholsyndrom 149

$12.1 \quad$ Einleitung $-\mathbf{1 4 9}$

12.2 Langzeitstudien in den USA, Frankreich, Finnland und

Deutschland 149 
12.3 Vorurteile und falsche Vorstellungen von einem Fetalen Alkoholsyndrom — 151

12.4 Untersuchungen bis ins Erwachsenenalter $-\mathbf{1 5 3}$

13 Klinik des Fetalen Alkoholsyndroms im Erwachsenenalter (FAS-adult) 167

$13.1 \quad$ Einleitung und ethische Überlegungen - 167

13.2 Diagnose mithilfe des 4-Digit Diagnostic Codes - 168

13.3 Sekundäre Störungen beim FAS des Erwachsenen - 177

13.4 Konflikte mit dem Gesetz $-\mathbf{1 8 0}$

14 Neuropsychologische Diagnostik - 183

14.1 Allgemeines - 183

14.2 Die neuropsychologische Testung - 183

14.3 Störungen der Intelligenz/kognitiven Leistungsfähigkeit - 185

14.4 Störung des Lernens und des Gedächtnisses - 185

14.5 Visuelle und räumlich-konstruktive Wahrnehmung - 186

$\mathbf{1 4 . 6}$ Motorik $\mathbf{1 8 7}$

14.7 Sprache $-\mathbf{1 8 8}$

14.8 Störungen des Verhaltens und sozialer Fertigkeiten - 188

14.9 Exekutive Funktionen - 189

14.10 Neuropsychologie des FASD im Erwachsenenalter - 194

Teil IV Intervention, Prävention und sozialrechtliche Aspekte

15 Therapeutische Aspekte und Interventionsmodelle - 197

$15.1 \quad$ Allgemeine Aspekte - 197

15.2 Interventionsmodelle $-\mathbf{1 9 8}$

16 Prävention von FAS und FASD - 203

$16.1 \quad$ Einleitung 203

16.2 Unspezifische, gesellschaftliche Suchtprävention — 204

16.3 Suchtprävention im Kindesalter - 204

16.4 Jugendliche und junge Erwachsene - 205

16.5 Erwachsene Frauen und Paare mit Kinderwunsch - 206

16.6 Spezifische Präventionskonzepte für Schwangere - 206

16.7 Erfassung von Risiken in der Schwangerenberatung - 207

16.8 Schwangere mit hohem Risiko für eine alkoholexponierte Schwangerschaft -208

17 FASD und sozialrechtliche Aspekte in der Praxis - 212 
xiv - Inhalt

Teil V 20 Lebensberichte über und von Patienten mit FASD

A Betreute Wohngemeinschaften und betreutes Einzelwohnen für Menschen mit FASD - 271

A.1 Die Wohngemeinschaften - FASD - 272

A.2 Das betreute Einzelwohnen - FASD — 273

B S3-Leitlinie zur Diagnostik des Fetalen Alkoholsyndroms -275

B.1 Einleitung $-\mathbf{2 7 5}$

B.2 Methodik 276

B.3 Kriterien für die Diagnose FAS bei Kindern und Jugendlichen - 278

Literatur $-\mathbf{2 8 9}$

Stichwortverzeichnis - $\mathbf{3 0 5}$

Namensverzeichnis -313

Einige Adressen in Deutschland mit spezieller Kenntnis in FASD-Diagnostik — 315 